\title{
Validación del Psychiatric Diagnostic Screening Questionnaire (PDSQ) en una muestra de pacientes alcohólicos españoles
}

\section{Validation of the Psychiatric Diagnostic Screening Questionnaire (PDSQ) in a Spanish sample of alcoholic patients}

\author{
Bartolomé Pérez Gálvez ${ }^{*}$, **; Lorena García \\ Fernández $^{* *}$; Ma Pura de Vicente Manzanaro*; \\ $M^{a}$ Angustias Oliveras ValenZuela ${ }^{* \star},{ }^{* \star \star}$.
}

$\mid \begin{aligned} & * \quad \text { Unidad de Alcohología. Hospital Universitario de San Juan (Alicante). } \\ & \text { ** } \begin{array}{l}\text { Departamento de Medicina Clínica. División de Psiquiatría. Universidad } \\ \text { Miguel Hernández. San Juan (Alicante). }\end{array} \\ & { }^{* \star *} \text { Servicio de Psiquiatría. Hospital Universitario de San Juan (Alicante). }\end{aligned}$

Enviar correspondencia a:

Bartolomé Pérez Gálvez

Hospital Universitario de San Juan. Servicio de Psiquiatría - Unidad de Alcohología. Ctra. Alicante-Valencia, s/n. 03550 - San Juan (Alicante)

Email: bperezgalvez@ono.com

\section{RESUMEN}

Objetivos: Validar el Psychiatric Diagnostic Screening Questionnaire (PDSO) en población española dependiente al alcohol, determinando su utilidad práctica como instrumento de detección de comorbilidad psiquiátrica.

Material y Método: El PDSO (Zimmerman, 2001) es un cuestionario autoadministrado de 125 items que solo requiere 15 minutos para su aplicación, permitiendo un rápido y eficaz cribaje de los trastornos psiquiátricos (Eje I) más comunes entre los adictos a sustancias, asi como de la dependencia al alcohol y a otras drogas.

Previa adaptación lingüistica (traducción/retrotraducción) y comprobada su validez de contenido, el PDSO fue administrado a 156 sujetos alcoholdependientes (criterios DSM-IV; mujeres $=36 \%$ ), atendidos consecutivamente en una Unidad de Alcohología. Se determinaron sus propiedades psicométricas (fiabilidad y validez), aplicándose criterios DSM-IV como patrón de referencia mediante entrevista SCID.

Resultados: Utilizando los puntos de corte originales, el PDS0 registró una excelente consistencia interna $(\alpha=0.68-0.96)$ y validez diagnóstica, con unos promedios de sensibilidad $=99.6 \%$ (rango: $96.6 \%-100 \%)$, especificidad $=69.5 \%$ (rango: $51.9 \%-94.6 \%$ ) y VPN $=99.8 \%$ (rango: $98.6 \%$ 100\%). La eficiencia diagnóstica media con los puntos de corte originales fue del $73.2 \%$, elevándose al $91.6 \%$ utilizando nuevos puntos de corte en determinadas escalas, con un grado de acuerdo con los criterios DSM-IV de $\kappa=.303-.896$.

Conclusión: EI PDSQ es un instrumento fiable y válido para detectar comorbilidad psiquiátrica en sujetos alcohólicos. Sus propiedades psicométricas y el valor añadido de su autoadministración y escaso consumo de tiempo permiten aconsejar su utilización en el ámbito clínico habitual.

Palabras clave: Alcoholismo, cuestionarios, diagnóstico dual, propiedades psicométricas, trastornos psiquiátricos. recibido: Diciembre 2009 aceptado: Febrero 2010

\section{ABSTRACT}

Objective: This study evaluates the psychometric properties of the Psychiatric Diagnostic Screening Questionnaire (PDSO) in alcohol-dependent Spanish population as a means of detecting psychiatric comorbidity.

Methods: The PDSO (Zimmerman, 2001) is a self-administered questionnaire with 125 items which can be applied in just 15 minutes, enabling rapid and effective screening of the psychiatric disorders (Axis I) most commonly found among substance abusers. After linguistic adaptation (translation/back-translation) and verification of its content validity, the PDSO was administered to 156 alcohol-dependent patients (DSM-IV criteria; women $=36 \%$ ), treated consecutively in an alcohol-disorders treatment setting. Its psychometric properties (reliability and validity) were determined, applying the DSM-IV criteria as a "gold standard", by means of SCID interview.

Results: Using the original cut-off points, the PDSO showed excellent internal reliability ( $\alpha=0.68-0.96)$ and diagnostic validity, with mean sensitivity $=99.6 \%$ (range: $96.6 \%-100 \%)$, specificity $=69.5 \%$ (range: $51.9 \%$ 94.6\%) and NPV $=99.8 \%$ (range: $98.6 \%-100 \%$ ). Diagnostic efficiency with the original cut-off points was $73.2 \%$, rising to $91.6 \%$ using new cut-off points for certain scales, with a degree of agreement with DSM-IV criteria of $\kappa=.303-.896$.

Conclusions: The PDSO is a reliable and valid instrument for detecting psychiatric comorbidity in alcoholic individuals. Its psychometric properties and the added value of self-administration and short application time make it a recommended instrument for use in routine clinical settings.

Key Words: Alcoholism, dual diagnosis, mental disorders, psychometric properties, questionnaires. 


\section{INTRODUCCIÓN}

L a detección de la comorbilidad psiquiátrica constituye una prioridad en la atención a la población adicta sustancias. En España se estima que el 53\% de los pacientes atendidos tanto en centros específicos de drogodependencias como de Salud Mental presentarian un trastorno dual. De estos, dos terceras partes cumplen los criterios diagnósticos de dos o más trastornos mentales ${ }^{1}$. Entre la población que solicita tratamiento en centros específicos de drogodependencias, los adictos al alcohol registran la prevalencia más elevada, alcanzando un $48.5 \%$ cuando se asocia a un trastorno adictivo a otra sustancia ${ }^{2}$.

En la práctica clínica, la elevada comorbilidad psiquiátrica entre los pacientes drogodependientes obliga a establecer un diagnóstico fiable de la misma. Sin embargo, éste suele ser complejo por la confusión que generan los efectos agudos y crónicos de las sustancias, así como la sintomatología de abstinencia, obligando a establecer la diferenciación entre trastornos primarios e inducidos ${ }^{3}$. Las distintas entrevistas diagnósticas disponibles facilitan la fiabilidad de estos diagnósticos. Entre las entrevistas validadas y adaptadas en español, cabe reseñar la Psychiatric Research Interview for Substance and Mental Disorders (PRISM) ${ }^{4}$. la MiniInternational Neuropsychiatric Interview $(\mathrm{MINI})^{5}$ o la más reciente Diagnostic Interview for Genetic Studies (DIGS) ${ }^{6}$. A pesar de su contrastada utilidad y eficacia diagnóstica, estas entrevistas requieren un tiempo excesivo para su aplicación en la práctica clínica habitual.

Aunque ciertamente no pueden alcanzar el mismo grado de fiabilidad que las entrevistas diagnósticas, algunos cuestionarios son de especial utilidad en la detección de la comorbilidad psiquiátrica. Entre este tipo de instrumentos destaca el Psychiatric Diagnostic Screening Questionnaire (PDSO) $)^{7,8}$ que permite una detección fiable de los trastornos psiquiátricos de Eje I más prevalentes en la población drogodependiente. El hecho de que apenas requiera 15-20 minutos para su aplicación así como su diseño autoadministrado con entrevista diagnóstica posterior, facilitan considerablemente su utilización en escenarios clínicos con limitaciones de tiempo.

Tanto por la necesidad de disponer de un instrumento breve de cribaje psiquiátrico, como por la oportunidad que ofrece el PDSO para cubrir esta carencia, la presente investigación se dirige a adaptar y validar este cuestionario en una muestra clínica española de pacientes alcohólicos, determinando sus propiedades psicométricas de fiabilidad y validez diagnóstica.

\section{MÉTODO}

\section{Participantes}

Realizamos un estudio observacional dirigido a validar el Psychiatric Diagnostic Screening Questionnaire (PDSO) en sujetos dependientes al alcohol que reciben tratamiento ambulatorio en una unidad especializada del sistema sanitario público. Como patrón de referencia se utilizaron los diagnósticos obtenidos mediante la entrevista SCID (criterios DSM-IV). La muestra está compuesta por 156 sujetos (100 hombres y 56 mujeres) atendidos consecutivamente en una unidad ambulatoria de alcohología y diagnosticados de Trastorno por Dependencia al Alcohol según criterios DSM-IV (criterio de inclusión). Debe reseñarse que la ratio hombre:mujer (2:1) fue atípica en el periodo en el que se realizó la validación del PDSO (septiembre de 2007 a mayo de 2008), si bien ha facilitado una mayor representatividad de mujeres dependientes al alcohol. Generalmente, esta ratio es de tres hombres por cada mujer atendida en la unidad asistencial de la que procede la muestra. Los criterios de exclusión comprendieron todas aquellas situaciones que dificultaran o imposibilitaran la correcta comprensión y respuesta del PDSO, tales como limitaciones visuales, de inteligencia o deterioro cognitivo severo. Ningún participante en el estudio fue excluido por estos motivos. Después de haber sido informados sobre los objetivos del estudio, todos los participantes aceptaron voluntariamente su inclusión en éste, sin que se registrara ningún caso de rechazo a participar en el mismo.

La edad media de la muestra fue de 41.61 años (d.s. = 8.09 años) con un rango de 22 a 66 años, no apreciándose diferencias significativas entre ambos sexos (hombres: 42.15 , d.s. $=8.59$ años; mujeres: 40.64 , d.s = 7.08 años; $t=1.117$; $p=.266$ ). Todos ellos eran de raza blanca y nacionalidad española.

\section{Instrumento y procedimiento}

El Psychiatric Diagnostic Screening Questionnaire (PDSO) es un cuestionario autoadministrado, constituido por 125 items con respuesta dicotómica ( $\mathrm{Si}=1$; No $=0$ ) y diseñado para la detección de 13 de los trastornos psiquiátricos de mayor prevalencia: Trastorno Depresivo Mayor (TDM), Trastorno por Estrés Postraumático (TEPT), Bulimia (BUL), Trastorno Obsesivo-Compulsivo (TOC), Crisis de Angustia (PAN), Psicosis (PSI), Agorafobia (AGO), Fobia Social (FOB), Abuso/Dependencia al Alcohol (ALC), Abuso/Dependencia de Sustancias (DRO), Trastorno de Ansiedad Generalizada (TAG), Trastorno de Somatización (SOM) e Hipocondría (HIP).

EI PDSO interroga al sujeto sobre la presencia de síntomas en dos períodos distintos de tiempo: en las últimas dos semanas, cuando se trata de las seis primeras subescalas (TDM, TEPT, BUL, TOC, PAN y PSI), o en los últimos seis meses, cuando se hace referencia a las otras siete patologías incluidas en el PDSO. En este último caso, el periodo cronológico se incrementa por cuanto los síntomas de estos trastornos pueden presentarse de forma intermitente y no ser evidentes en las dos semanas previas a la evaluación. Por otra parte, en algunas patologías como el TAG o la hipocondría, los criterios DSM-IV establecen una duración mínima de seis meses para poder establecer el diagnóstico.

En los estudios publicados hasta la fecha, el PDSO ha evidenciado una excelente consistencia interna en sus distintas subescalas, con un promedio del coeficiente $\alpha$ de 
Cronbach de 0.86 y una correlación media test-retest de $r=0.83$. Tratándose de un instrumento de cribaje y no de diagnóstico, Zimmerman y Mattia ${ }^{9}$ aconsejan establecer puntos de corte que correspondan a una sensibilidad $\geq 90 \%$ para obtener el menor número posible de falsos negativos. Con los puntos de cortes propuestos por los autores, las distintas escalas del PDSO registran un Valor Predictivo Negativo medio del $97 \%$ en su aplicación en población psiquiátrica.

\section{Proceso de traducción y adaptación lingüística}

Se realizó un proceso de traducción inglés-español y posterior retrotraducción español-inglés, verificando la igualdad de contenidos entre el original y la traducción final, así como la adecuada comprensión de sus ítems. En primer lugar el texto inglés fue traducido al español por un profesional bilingüe. A continuación, otra persona bilingüe retro-tradujo el texto español al inglés. Posteriormente, un tercer colaborador, igualmente bilingüe y de lengua nativa inglesa, comparó los dos textos (inicial y retrotraducción al inglés) confirmando la similitud de los contenidos, entendiéndose que la traducción al español correspondia fielmente con el texto original inglés.

El texto final en español fue valorado posteriormente por cinco personas de habla española y no integrantes de la muestra, al objeto de asegurar la correcta comprensión de los ítems. Concluida esta última comprobación, no fue preciso realizar nuevas correcciones en el cuestionario.

\section{Aplicación de los instrumentos}

Los sujetos participantes en el estudio completaron el PDSO como parte de la evaluación psicopatológica en su tratamiento. La administración del cuestionario se realizó transcurridas entre 4 y 6 semanas desde el inicio de la abstinencia, al objeto de evitar el sesgo producido por el consumo de alcohol.

La cumplimentación del cuestionario se realizó de forma autoadministrada, con un tiempo medio de aplicación de 18 minutos y 49 segundos (d.s. = 5' 44"). Cuando el cuestionario se entregaba al entrevistador, y antes de ser corregido, el paciente era evaluado mediante la entrevista diagnóstica SCID para cada uno de los diagnósticos incluidos en el PDSO. En consecuencia, el entrevistador era ciego a los resultados del PDSO en el momento en que se realizaba la entrevista.

\section{Análisis estadístico}

La consistencia interna de las distintas subescalas del PDSO fue analizada mediante el coeficiente $\alpha$ de Cronbach. La validez diagnóstica de cada subescala fue evaluada en términos de sensibilidad, especificidad, Valor Pronóstico Positivo (VPP), Valor Pronóstico Negativo (VPN) y eficiencia. Al objeto de anular el efecto del azar, se determinó el grado de concordancia (coeficiente Kappa de Cohen) con relación al patrón de referencia diagnóstico (criterios DSMIV). El análisis estadístico se realizó con el programa SPSS versión 15.0 para Windows, estableciéndose la significación estadística bilateral en $\mathrm{p} \leq .05$.

\section{RESULTADOS}

La Tabla 1 muestra las prevalencias diagnósticas registradas en la muestra de población estudiada, obtenidas mediante entrevista SCID. Los diagnósticos de TDM (34\%), PAN (18.6\%), TEPT (17.9\%), FOB (17.3\%) y AGO (16\%) fueron los más representados. Un $26.3 \%$ de los sujetos cumplieron criterios de dependencia a otras sustancias (cannabis y/o cocaína).

Tabla 1: Prevalencia de patología psiquiátrica en la muestra $(n=156 ;$ g.l. $=1)$, evaluada mediante SCID.

\begin{tabular}{|c|c|c|c|c|c|c|}
\hline & Hombres & Mujeres & $x^{2}$ & $\mathrm{p}$ & Total & $\mathrm{n}$ \\
\hline TDM & $25.0 \%$ & $50.0 \%$ & 10.002 & 0.002 & $34.0 \%$ & 53 \\
\hline TEPT & $12.0 \%$ & $28.6 \%$ & 6.694 & 0.010 & $17.9 \%$ & 28 \\
\hline BUL & $1.0 \%$ & $12.5 \%$ & 9.758 & 0.002 & $5.1 \%$ & 8 \\
\hline TOC & $12.0 \%$ & $5.4 \%$ & 1.823 & 0.177 & $9.6 \%$ & 15 \\
\hline PAN & $14.0 \%$ & $26.8 \%$ & $3 . .878$ & 0.049 & $18.6 \%$ & 29 \\
\hline PSI & $2.0 \%$ & $1.8 \%$ & 0.090 & 0.926 & $1.9 \%$ & 3 \\
\hline AGO & $15.0 \%$ & $17.9 \%$ & 0.218 & 0.641 & $16.0 \%$ & 25 \\
\hline FOB & $13.0 \%$ & $25.0 \%$ & 3.612 & 0.057 & $17.3 \%$ & 27 \\
\hline DRO & $22.0 \%$ & $33.9 \%$ & 2.636 & 0.104 & $26.3 \%$ & 41 \\
\hline TAG & $8.0 \%$ & $10.7 \%$ & 0.324 & 0.569 & $9.0 \%$ & 14 \\
\hline SOM & $2.0 \%$ & $8.9 \%$ & 4.021 & 0.045 & $4.5 \%$ & 7 \\
\hline HIP & $5.0 \%$ & $3.6 \%$ & 0.171 & 0.679 & $4.5 \%$ & 7 \\
\hline
\end{tabular}


La consistencia interna de las distintas escalas del PDSO osciló en el rango $\alpha=0.68-0.96$, con un promedio de $\alpha=0.86$ (Tabla 2). Los seis ítems que evalúan la ideación autolítica ( $n^{\circ} 16$ a 21 ) registraron igualmente una elevada consistencia interna $(\alpha=0.89)$.

Tabla 2: Consistencia interna.

\begin{tabular}{cc}
\hline & $\alpha$ \\
\hline TDM & 0.89 \\
TEPT & 0.92 \\
BUL & 0.91 \\
TOC & 0.79 \\
PAN & 0.91 \\
PSI & 0.68 \\
PAN & 0.84 \\
FOB & 0.88 \\
DRO & 0.96 \\
TAG & 0.89 \\
SOM & 0.79 \\
HIP & 0.86 \\
\hline Promedio & 0.86 \\
\hline
\end{tabular}

En la Tabla 3 se refleja la validez diagnóstica de cada una de las subescalas del PDSO. Utilizando los puntos de corte propuestos por Zimmerman ${ }^{8}$ las subescalas del PDSO registraron una sensibilidad media del 99.6\% (rango: 96.6\%-100\%) y VPN del 99.8\% (rango: 98.6\%-100\%). La especificidad fue sensiblemente inferior, con un promedio de 69.5\% (rango: $51.9 \%-94.6 \%$ ) y ocho subescalas registrando una especificidad inferior al 70\%. Tampoco fue elevada la eficiencia diagnóstica media (73.18\%), siendo inferior al $80 \%$ en ocho de las doce subescalas. De igual modo, en ocho subescalas y utilizando los puntos de corte originales, el grado de concordancia con los criterios diagnósticos DSM-IV no superó el valor de $\kappa=0.60$ propuesto por Landis y Koch ${ }^{10}$ como límite para ser considerado como bueno.

Es preciso advertir que los puntos de corte propuestos por Zimmerman se dirigen a obtener el menor número posible de falsos negativos. Bajo este criterio es comprensible que se registren valores elevados de sensibilidad y VPN, pero con una considerable disminución en la especificidad, VPP y eficiencia diagnóstica de la prueba.

Dadas las diferencias de las características de la muestra respecto a las incluidas en estudios previos, procedimos a ajustar los puntos de corte bajo el criterio de mantener

Tabla 3: Validez diagnóstica del PDSO.

\begin{tabular}{|c|c|c|c|c|c|c|}
\hline Escala & Punto de corte & Especificidad & Sensibilidad & VPN & VPP & Eficiencia \\
\hline \multirow[t]{2}{*}{ TDM } & 9 & $68.9 \%$ & $98.1 \%$ & $98.6 \%$ & $61.9 \%$ & $78.8 \%$ \\
\hline & $11^{*}$ & $81.6 \%$ & $92.5 \%$ & $95.5 \%$ & $72.1 \%$ & $85.3 \%$ \\
\hline \multirow[t]{2}{*}{ TEPT } & 5 & $67.2 \%$ & $100.0 \%$ & $100.0 \%$ & $40.0 \%$ & $73.1 \%$ \\
\hline & $10^{\circ}$ & $95.3 \%$ & $96.4 \%$ & $99.2 \%$ & $81.8 \%$ & $95.5 \%$ \\
\hline BUL & 7 & $94.6 \%$ & $100.0 \%$ & $100.0 \%$ & $50.0 \%$ & $94.9 \%$ \\
\hline \multirow[t]{2}{*}{ TOC } & 1 & $57.4 \%$ & $100.0 \%$ & $100.0 \%$ & $20.0 \%$ & $61.5 \%$ \\
\hline & $3^{*}$ & $89.4 \%$ & $100.0 \%$ & $100.0 \%$ & $50.0 \%$ & $90.4 \%$ \\
\hline \multirow[t]{2}{*}{ PAN } & 4 & $86.6 \%$ & $96.6 \%$ & $99.1 \%$ & $62.2 \%$ & $88.5 \%$ \\
\hline & $6^{*}$ & $96.9 \%$ & $96.4 \%$ & $99.2 \%$ & $87.1 \%$ & $96.8 \%$ \\
\hline \multirow[t]{2}{*}{ PSI } & 1 & $58.2 \%$ & $100.0 \%$ & $100.0 \%$ & $4.5 \%$ & $59.0 \%$ \\
\hline & $5^{*}$ & $98.7 \%$ & $66.7 \%$ & $99.3 \%$ & $50.0 \%$ & $98.1 \%$ \\
\hline \multirow[t]{2}{*}{ AGO } & 4 & $76.3 \%$ & $100.0 \%$ & $100.0 \%$ & $44.6 \%$ & $80.1 \%$ \\
\hline & $6^{*}$ & $93.1 \%$ & $92.0 \%$ & $98.4 \%$ & $71.9 \%$ & $93.0 \%$ \\
\hline \multirow[t]{2}{*}{ FOB } & 4 & $51.9 \%$ & $100.0 \%$ & $100.0 \%$ & $30.3 \%$ & $60.3 \%$ \\
\hline & $9^{*}$ & $96.1 \%$ & $92.6 \%$ & $98.4 \%$ & $83.3 \%$ & $95.5 \%$ \\
\hline DRO & 1 & $91.3 \%$ & $100.0 \%$ & $100.0 \%$ & $80.4 \%$ & $93.6 \%$ \\
\hline \multirow[t]{2}{*}{ TAG } & 7 & $54.2 \%$ & $100.0 \%$ & $100.0 \%$ & $17.7 \%$ & $58.3 \%$ \\
\hline & $9^{*}$ & $73.2 \%$ & $100.0 \%$ & $100.0 \%$ & $26.9 \%$ & $75.6 \%$ \\
\hline \multirow[t]{2}{*}{ SOM } & 2 & $59.7 \%$ & $100.0 \%$ & $100.0 \%$ & $10.4 \%$ & $61.5 \%$ \\
\hline & $5^{\circ}$ & $95.3 \%$ & $85.7 \%$ & $99.3 \%$ & $46.2 \%$ & $94.9 \%$ \\
\hline \multirow[t]{2}{*}{ HIP } & 1 & $67.1 \%$ & $100.0 \%$ & $100.0 \%$ & $12.5 \%$ & $68.6 \%$ \\
\hline & $3^{*}$ & $85.9 \%$ & $85.7 \%$ & $99.2 \%$ & $22.2 \%$ & $85.9 \%$ \\
\hline
\end{tabular}

'Se utilizan los puntos de corte obtenidos en la versión original del PDSO (Zimmerman, 2002). Los señalados con asterisco corresponden a los propuestos en el presente estudio para incrementar la eficiencia diagnóstica. 
Tabla 4: Prevalencia diagnóstica de acuerdo a la SCID y PDSO.

\begin{tabular}{|c|c|c|c|c|}
\hline Escala & Punto de corte & SCID & PDSO & $\kappa$ \\
\hline \multirow[t]{2}{*}{ TDM } & 9 & $34.0 \%$ & $53.8 \%$ & 0.587 \\
\hline & $11^{*}$ & $34.0 \%$ & $43.6 \%$ & 0.692 \\
\hline \multirow[t]{2}{*}{ TEPT } & 5 & $17.9 \%$ & $44.9 \%$ & 0.424 \\
\hline & $10^{*}$ & $17.9 \%$ & $21.2 \%$ & 0.817 \\
\hline BUL & 7 & $5.1 \%$ & $10.3 \%$ & 0.642 \\
\hline \multirow[t]{2}{*}{ TOC } & 1 & $9.6 \%$ & $48.1 \%$ & 0.206 \\
\hline & 3 & $9.6 \%$ & $19.2 \%$ & 0.618 \\
\hline \multirow[t]{2}{*}{ PAN } & 4 & $18.6 \%$ & $28.8 \%$ & 0.686 \\
\hline & $6^{*}$ & $18.6 \%$ & $19.9 \%$ & 0.896 \\
\hline \multirow[t]{2}{*}{ PSI } & 1 & $1.9 \%$ & $42.9 \%$ & 0.051 \\
\hline & $5^{\circ}$ & $1.9 \%$ & $2.6 \%$ & 0.562 \\
\hline \multirow[t]{2}{*}{ AGO } & 4 & $16.0 \%$ & $35.9 \%$ & 0.508 \\
\hline & $6^{*}$ & $16.0 \%$ & $20.5 \%$ & 0.765 \\
\hline \multirow[t]{2}{*}{ FOB } & 4 & $17.3 \%$ & $57.1 \%$ & 0.272 \\
\hline & $9^{\circ}$ & $17.3 \%$ & $19.2 \%$ & 0.850 \\
\hline DRO & 1 & $26.3 \%$ & $32.7 \%$ & 0.847 \\
\hline \multirow[t]{2}{*}{ TAG } & 7 & $9.0 \%$ & $50.6 \%$ & 0.175 \\
\hline & $9^{\circ}$ & $9.0 \%$ & $33.3 \%$ & 0.329 \\
\hline \multirow[t]{2}{*}{ SOM } & 2 & $4.5 \%$ & $42.9 \%$ & 0.117 \\
\hline & $5^{\circ}$ & $4.5 \%$ & $8.3 \%$ & 0.575 \\
\hline \multirow[t]{2}{*}{ HIP } & 1 & $4.5 \%$ & $35.9 \%$ & 0.155 \\
\hline & $3^{\circ}$ & $4.5 \%$ & $17.3 \%$ & 0.303 \\
\hline
\end{tabular}

"Puntos de corte modificados para incrementar la eficiencia diagnóstica.

una sensibilidad elevada y un VPN próximo al 100\%, que permitieran la utilización del PDSO en el cribaje diagnóstico. Con estos ajustes, la eficiencia diagnóstica del PDSO se incrementó sustancialmente (ver Tabla 3). Los puntos de corte seleccionados para la muestra evidenciaron una excelente validez predictiva en once subescalas. El considerable incremento obtenido en la especificidad media (91\%) y la eficiencia diagnóstica $(91.6 \%)$ compensó la ligera disminución registrada en la sensibilidad (92.3\%) y VPN (99\%). Un dato relevante fue el hecho de que, con los nuevos puntos de corte, el coeficiente $\kappa$ de Cohen fue superior a 0.60 en ocho subescalas y solo en TAG e HIP se registraron débiles concordancias con los criterios DSM-IV $(\kappa \leq 0.40)$.

Las distintas prevalencias registradas en la muestra, utilizando el PDSO y la SCID, así como sus grados de concordancia aparecen reflejadas en la Tabla 4.

\section{DISCUSIÓN}

El presente trabajo se dirige a contrastar la utilidad del Psychiatric Diagnostic Screening Questionnaire (PDSO) en el cribaje de la comorbilidad psiquiátrica en pacientes ambulatorios alcohólicos. Se trata de la adaptación y validación de este instrumento en lengua española y, a la vez, del primer estudio realizado con este cuestionario específicamente en población clínica dependiente al alcohol. Las propiedades psicométricas del PDSO así como el escaso tiempo necesario para su aplicación, han evidenciado la utilidad de este instrumento para la detección de comorbilidad psiquiátrica en este tipo de pacientes, en el contexto de la práctica clínica habitual. Sin embargo, los puntos de corte propuestos por Zimmerman ${ }^{8}$ en diez subescalas no presentaron buenas propiedades psicométricas en la muestra evaluada, razón por las que fueron modificados al objeto de incrementar la eficiencia diagnóstica del instrumento. En consecuencia, es aconsejable que estos nuevos puntos de corte sean confirmados en estudios posteriores que sean realizados con muestras clínicas de similares características.

Como primera propiedad psicométrica evaluada, la consistencia interna del PDSO coincide con la comunicada por Zimmerman y Mattia ${ }^{11}$ ratificando la adecuada fiabilidad del instrumento. Con un promedio de $\alpha=0.86$, las distintas subescalas del cuestionario registraron una fiabilidad entre buena y excelente. La excepción fue la subescala de Psicoticismo, con un débil coeficiente de Cronbach ( $\alpha=$ 0.68). Este resultado coincide con el observado por los autores del PDSO, quienes obtuvieron una $\alpha=0.66$ para esta subescala. No ocurre lo mismo con la correspondiente al Trastorno por Somatización (SOM), de consistencia débil en el estudio original ( $\alpha=0.66)$ y que en nuestro análisis alcanza un aceptable nivel de fiabilidad ( $\alpha=0.79$ ). En consecuencia, la fiabilidad de las subescalas del PDSO no parece estar afectada por la distinta naturaleza de las muestras estudiadas hasta la fecha, ofreciendo una constatada estabilidad de esta propiedad psicométrica.

Confirmada su fiabilidad, se comprobó la validez diagnóstica del instrumento. EI PDSO es un instrumento diseñado para ser utilizado en detección y valoración inicial en la práctica clínica $y$, por tanto, pretende evitar el mayor número posible de falsos negativos. Como 
señalan Rush, Zaslavska y Veldhuizen ${ }^{12}$ el objetivo de un instrumento de cribaje es detectar señales de alarma o "red flags" que orienten hacia la necesidad de realizar una evaluación posterior más detallada. Estos instrumentos deben caracterizarse por una sensibilidad y valor predictivo negativo elevado, de tal modo que permitan detectar el mayor número de casos probables y aporten la seguridad de que un caso negativo realmente lo es. Para ello, Zimmerman y Mattia ${ }^{9,11}$ recomiendan puntos de corte que permitan alcanzar una sensibilidad del 90\%. Cabe recordar que una alta sensibilidad suele acompañarse por la disminución en la especificidad del instrumento.

Con los puntos de corte originales, el PDSO ha registrado una moderada eficiencia diagnóstica, clasificando correctamente al $73.2 \%$ de los sujetos si bien este valor se incrementa hasta un $91.6 \%$ cuando se modifican los puntos de corte en determinadas patologías. Sin embargo, y dada su naturaleza de instrumento de cribaje en la práctica clínica, el principal parámetro de validez diagnóstica del PDSO debe ser su valor predictivo negativo (VPN). Los resultados obtenidos en nuestro estudio ofrecen un elevado grado de coincidencia con los comunicados en la validación original del cuestionario. En términos globales, el PDSO registró un VPN de $99.8 \%$ utilizando los puntos de corte originales, y de $99 \%$ para los puntos de corte modificados, en ambos casos muy elevados y ligeramente superiores al $97.1 \%$ obtenido inicialmente por Zimmerman y Mattia ${ }^{9}$. Solo en la escala TDM se registraron diferencias de interés, con un VPN sensiblemente más elevado en nuestra muestra (95.5\% frente a 86\%). Esta diferencia concuerda con la registrada en dos estudios realizados con población adicta ${ }^{13,14}$ en los que se obtuvo un VPN de $97 \%$ para esta escala, más próximo al obtenido en nuestra validación. A la vista de cuanto antecede se refrenda la elevada fiabilidad del PDSO para evitar falsos negativos.

Los análisis de sensibilidad y especificidad de la prueba también ofrecen positivos resultados que corroboran la utilidad del PDSO en la población dependiente al alcohol. Respecto a la primera -sensibilidad- es evidente que supera holgadamente el 90\% mínimo que aconsejaban Zimmerman y Mattia ${ }^{9}$ para su utilización en la práctica clínica. De hecho, se obtienen valores extremos que mejoran el promedio de $88.25 \%$ registrado en la validación original. Obviamente, los valores no son tan favorables en cuanto a la especificidad como resultado de unos bajos puntos de corte que permitan evitar falsos negativos. Similares resultados fueron obtenidos por Castel et al. ${ }^{14}$. quienes recuerdan que el cuestionario fue inicialmente validado utilizando una muestra de población psiquiátrica, con características distintas a los pacientes adictos a sustancias. En nuestro caso y utilizando los puntos de corte originales, la especificidad media fue aceptable e, incluso, ligeramente más elevada que en la validación original del cuestionario (69.47\% frente a 67.83\%). Como advierten Castel y colaboradores ${ }^{14}$, el hecho de que la muestra de validación original fuera de características distintas a la población adicta a sustancias -se utilizó población clínica psiquiátrica- justificaría este tipo de diferencias.
La validez predictiva de un instrumento de screening puede variar dependiendo de las características de la población en la que se aplique. Hasta la fecha, solo tres estudios han estudiado las características psicométricas del PDSO en población drogodependiente $\mathrm{e}^{13-15}$, habiéndose constatado ligeras variaciones en la validez predictiva de algunas subescalas. Los antecedentes existentes suelen excluir cinco escalas (BUL, PSI, DRO, SOM e HIP), además de la correspondiente al abuso/dependencia al alcohol (ALC). También debe considerarse que estas investigaciones no son homogéneas en la composición de la muestra de población estudiada -en relación al sexo y a la sustancia adictiva-, dificultando aún más su comparación.

Restringiendo nuestra comparación de resultados a los estudios realizados en población adicta a drogas observamos mayor nivel de concordancia en la validez diagnóstica del PDSO. Un hallazgo común es el hecho de que los adictos a sustancias suelen presentar una sensibilidad ligeramente más elevada que la población psiquiátrica. La sensibilidad media obtenida en nuestro estudio - para las patologías incluidas en la comparación y con los puntos de corte originales- fue del 99\%, próximo a los valores de $92 \%$ y $96 \%$ registrados por otros autores ${ }^{14-15}$. En relación a la especificidad, los resultados obtenidos con los mismos criterios (66\%) también son similares a los comunicados por Zimmerman ${ }^{15}$ así como por Magruder et al. ${ }^{13}$ ( $63 \%$ y $69 \%$, respectivamente). En consecuencia, objetivamos ligeras diferencias respecto a los escasos antecedentes publicados, diferencias que pudieran estar motivadas por las distintas composiciones de las muestras de población utilizadas en cada estudio.

Por otra parte se ha objetivado que la validez predictiva del PDSO parece estar influenciada por el periodo de tiempo estudiado en cada subescala. En nuestro estudio, la administración del PDSO se realizó entre 30 y 45 dias después de haberse iniciado la abstinencia En consecuencia, los síntomas evaluados en un período superior pueden mostrar cierta "contaminación" por el uso de esta sustancia. Los resultados obtenidos apuntan en esta dirección, observándose coeficientes $\kappa$ más elevados en aquellos trastornos que son evaluados en base a la presencia de sintomas en las dos semanas previas a la administración del cuestionario - período en el que los sujetos evaluados no consumian alcohol- en contraposición a algunas patologias en las que el PDSO evalúa la sintomatología presente en los seis meses anteriores, cuando los pacientes aún no habian iniciado la abstinencia. Tal vez por ello, el trastorno por ansiedad generalizada o los dos trastornos somatomorfos incluidos en el PDSO (somatización e hipocondria) registraron pobres concordancias diagnósticas con los criterios DSM-IV. En consecuencia, cabe esperar una mayor fiabilidad diagnóstica demorando la administración del PDSO durante un periodo de tiempo adecuado. No obstante, en el ámbito clínico no parece aconsejable demorar en exceso el diagnóstico psiquiátrico, siendo adecuado establecer un prudente "periodo ventana" que evite el factor de confusión introducido por el consumo y, al mismo tiempo, no retrase indebidamente el diagnóstico e inicio del oportuno tratamiento. 
A nuestro juicio, una limitación que todavía presenta el PDSO es el reducido número de estudios publicados referidos a su aplicación en muestras de pacientes drogodependientes. Sin embargo se trata de un instrumento ampliamente extendido, como lo demuestra su utilización en ensayos clínicos de gran tamaño muestral como el STAR-D ${ }^{16-18}$. Esta limitación aconseja su replicación en futuras investigaciones, al objeto de disponer de muestras más elevadas y homogéneas que sustenten los datos disponibles en la actualidad.

A pesar de estas observaciones, el PDSO puede ser considerado como un fiable y válido instrumento de cribaje de comorbilidad psiquiátrica en sujetos adictos a sustancias, con el valor añadido de su autoadministración y escaso consumo de tiempo. Debemos insistir en su condición de instrumento de cribado que no exime de la necesidad de efectuar un correcto diagnóstico confirmatorio. En conclusión, las propiedades psicométricas del PDSO refrendan una adecuada fiabilidad y validez en la detección de casos psiquiátricos - una vez corregidos los puntos de corte en distintas subescalas del cuestionario-, cumpliendo exitosamente el objetivo para el que fue diseñado.

\section{AGRADECIMIENTOS}

Los autores desean agradecer al Dr. Mark Zimmerman su aprobación para validar el PDSO en población española.

Este artículo se ha realizado en el contexto de la Red de Trastornos Adictivos, Red RTA, RD06/0001/0008. Redes Temáticas de Investigación Cooperativa en Salud, Ministerio de Sanidad y Consumo, Instituto de Salud Carlos III.

\section{REFERENCIAS}

1. Asociación Española de Patología Dual. Estudio epidemiológico para determinar la prevalencia, diagnóstico y actitud terapéutica de la patología dual en la Comunidad de Madrid. Madrid; 2008.

2. Gual A. Dual diagnosis in Spain. Drug Alcohol Rev 2007; 26: 65-71.

3. Torrens M, Martín-Santos R, Samet S. Importance of clinical diagnoses for comorbidity studies in substance use disorders. Neurotox Res 2006; 10: 253-61.

4. Torrens M, Serrano D, Astals M, Pérez-Dominguez G, MartínSantos R. Diagnosing comorbid psychiatric disorders in substance abusers: validity of the Spanish versions of the Psychiatric Research Interview for Substance and Mental Disorders and the Structured Clinical Interview for DSM-IV. Am J Psychiatry 2004; 161: 1231-7.

5. Ferrando L, Bobes J, Gibert J, Soto M, Soto O. Mini-International Neuropsychiatric Interview. Versión en Español 5.0.0. 2005. Disponible en: https://www.medical-outcomes.com/HTMLFiles/ MINI/MINI.htm [consultado el 21/11/09].

6. Roca M, Martín-Santos R, Sáiz J, Obiols J, Serrano MJ, Torrens M et al. Diagnostic Interview for Genetic Studies (DIGS): inter-rater and test-retest reliability and validity in a Spanish population. Eur Psychiatry 2007; 22: 44-8.
7. Zimmerman M, Mattia Jl. The reliability and validity of a screening Questionnaire for 13 DSM-IV Axis I disorders (the Psychiatric Diagnostic Screening Questionnaire) in psychiatric outpatients. J Clin Psychiatry 1999; 60: 677-83.

8. Zimmerman M. The Psychiatric Diagnostic Screening Questionnaire manual. Los Angeles (CA): Western Psychological Services; 2002.

9. Zimmerman M, Mattia JI. A Self-Report Scale to Help Make Psychiatric Diagnoses: The Psychiatric Diagnostic Screening Questionnaire. Arch Gen Psychiatry 2001; 58: 787-94.

10. Landis J, Koch G. The measurement of observer agreement for categorical data. Biometrics 1977; 33: 159-74.

11. Zimmerman M, Mattia Jl. The Psychiatric Diagnostic Screening Questionnaire: Development, reliability and validity. Compr Psychiatry 2001; 42: 175-89.

12. Rush B, Zaslavska N, Veldhuizen S. A Comparison of alternative approaches for screening for substance abuse in two community mental health services. Toronto: Centre for Addiction and Mental Health. Health Systems Research \& Consulting Unit; 2005.

13. Magruder KM, Sonne SC, Brady KT, Quello S, Martin RH. Screening for co-occurring mental disorders in drug treatment populations. J Drug Issues 2005; 35: 593-605.

14. Castel S, Rush B, Kennedy S, Fulton K, Toneatto T. Screening for mental health problems among patients with substance use disorders: preliminary findings on the validation of a selfassessment instrument. Can J Psychiatry 2007; 52: 22-7.

15. Zimmerman $M$, Sheeran $T$, Chelminski I, Young D, Screening for psychiatric disorders in outpatients with DSM-IV substance use disorders. J Subst Abuse Treat 2004; 26: 181-8.

16. Rush AJ, Zimmerman M, Wisniewski SR, Fava M, Hollon SD, Warden D et al. Comorbid psychiatric disorders in depressed outpatients: Demographic and clinical features. J Affect Disord 2005; 87: 43-55.

17. Fava M, Rush AJ, Alpert JE, Carmin CN, Balasubramani GK, Wisniewski SR et al. What clinical and symptom features and comorbid disorders characterize outpatients with anxious major depressive disorder: a replication and extension. Can J Psychiatry 2006; 51: 823-35.

18. Howland RH, Rush AJ, Wisniewski SR, Trivedi MH, Warden D, Fava $\mathrm{M}$ et al. Concurrent anxiety and substance use disorders among outpatients with major depression: Clinical features and effect on treatment outcome. Drug Alcohol Depend 2009; 99: 248-60. 
